\title{
Factors associated with inter-institutional variations in sepsis rates of very-low-birth-weight infants in 34 Malaysian neonatal intensive care units
}

Nem-Yun $\underline{B o o}^{1}$, MBBS, FRCPCH, Irene Guat-Sim $\underline{\mathrm{Cheah}}^{2}$, MBBS, FRCPCH

INTRODUCTION This study aimed to determine whether patient loads, infant status on admission and treatment interventions were significantly associated with inter-institutional variations in sepsis rates in very-low-birth-weight (VLBW) infants in the Malaysian National Neonatal Registry (MNNR).

METHODS This was a retrospective study of $3,880 \mathrm{VLBW}(\leq 1,500 \mathrm{~g})$ infants admitted to 34 neonatal intensive care units (NICUs) in the MNNR. Sepsis was diagnosed in symptomatic infants with positive blood culture.

RESULTS Sepsis developed in 623 (16.1\%) infants; 61 (9.8\%) had early-onset sepsis (EOS) and 562 (90.2\%) had lateonset sepsis (LOS). The median EOS rate of all NICUs was 1.0\% (interquartile range [IQR] 0\%, 2.0\%). Compared with NICUs reporting no EOS $(n=14)$, NICUs reporting EOS $(n=20)$ had significantly higher patient loads (total live births, admissions, VLBW infants, outborns); more mothers with a history of abortions, and antenatal steroids and intrapartum antibiotic use; more infants requiring resuscitation procedures at birth; higher rates of surfactant therapy, pneumonia and insertion of central venous catheters. The median LOS rate of all NICUs was $14.5 \%$ (IQR $7.8 \%, 19.2 \%)$. Compared with NICUs with LOS rates below the first quartile $(n=8)$, those above the third quartile $(n=8)$ used less intrapartum antibiotics, and had significantly bigger and more mature infants, more outborns, as well as a higher number of sick infants requiring ventilator support and total parenteral nutrition.

CONCLUSION Patient loads, resuscitation at birth, status of infants on admission and treatment interventions were significantly associated with inter-institutional variations in sepsis.

Keywords: Malaysian, NICU, sepsis, VLBW infants

\section{INTRODUCTION}

Neonatal sepsis in very-low-birth-weight (VLBW) infants weighing $1,500 \mathrm{~g}$ or less is a common problem in neonatal intensive care units (NICUs), with high morbidity and mortality rates reported in the literature. ${ }^{(1-4)}$ Reports from various neonatal networks showed wide variations in sepsis rates $(8.5 \%-42.0 \%)$ among NICUs. ${ }^{(1,5-7)}$ For this study, data was collected from the Malaysian National Neonatal Registry (MNNR), which was established in 2003. One of the MNNR's aims was to improve standards of neonatal care among its NICUs through systematic learning from other units based on evidence. We aimed to determine whether differences in patient loads; demographic, perinatal and patient characteristics; and resuscitation measures at birth were significantly associated with inter-institutional variations in sepsis rates.

\section{METHODS}

This was a retrospective study using data from the MNNR, which had a membership of 34 NICUs from Malaysian government hospitals in 2010. The MNNR database was anonymised. Approval for the study was obtained from the National Institutes of Health Malaysia. The inclusion criteria were VLBW infants born between 1 January 2010 and 31 December 2010, either in MNNR hospitals (i.e. inborn) or other facilities (i.e. outborn), and who were admitted to NICUs at these hospitals. The demographic and clinical data of the infants was submitted to the MNNR by trained staff in a standard format. Each infant was a unique case that was not duplicated in the registry, irrespective of the number of times the infant had been admitted to different participating NICUs.

Sepsis was diagnosed in symptomatic infants with a positive blood or cerebrospinal culture. If the infant had confirmed sepsis, data was collected on whether the first episode of sepsis occurred on or before Day 3 of life (early-onset sepsis [EOS]), or after Day 3 of life (late-onset sepsis [LOS]). Data on the following organisms was collected: group B streptococcus (GBS), methicillin-resistant Staphylococcus aureus, coagulasenegative staphylococcus (CoNS), fungus, Staphylococcus aureus, Klebsiella spp., Pseudomonas spp., and Acinetobacter spp. Other organisms that were isolated were specified. Gestational assessment was based on the mother's last menstrual period, antenatal ultrasonography or Ballard's score. ${ }^{(8)}$ Infants were classified as appropriate for gestational age (AGA), small for gestational age and large for gestational age when their birth weights were between the 10th and 90th percentile, below the 10th percentile and above the 90th percentile for their gestational age, respectively. ${ }^{(9)}$ Intrapartum antibiotics were administered to the infants' mothers within 24 hours before delivery. Respiratory distress syndrome (RDS) was diagnosed based on clinical and

\footnotetext{
${ }^{1}$ Department of Population Health, Faculty of Medicine and Health Sciences, Universiti Tunku Abdul Rahman, Selangor, ${ }^{2}$ Department of Paediatrics, Paediatric Institute, Hospital Kuala Lumpur, Kuala Lumpur, Malaysia

Correspondence: Dr Nem-Yun Boo, Senior Professor, Department of Population Medicine, Faculty of Medicine and Health Sciences, Universiti Tunku Abdul Rahman, Jalan Sungai Long, Bandar Sungai Long, 43000 Kajang, Selangor, Malaysia. boony@utar.edu.my
} 


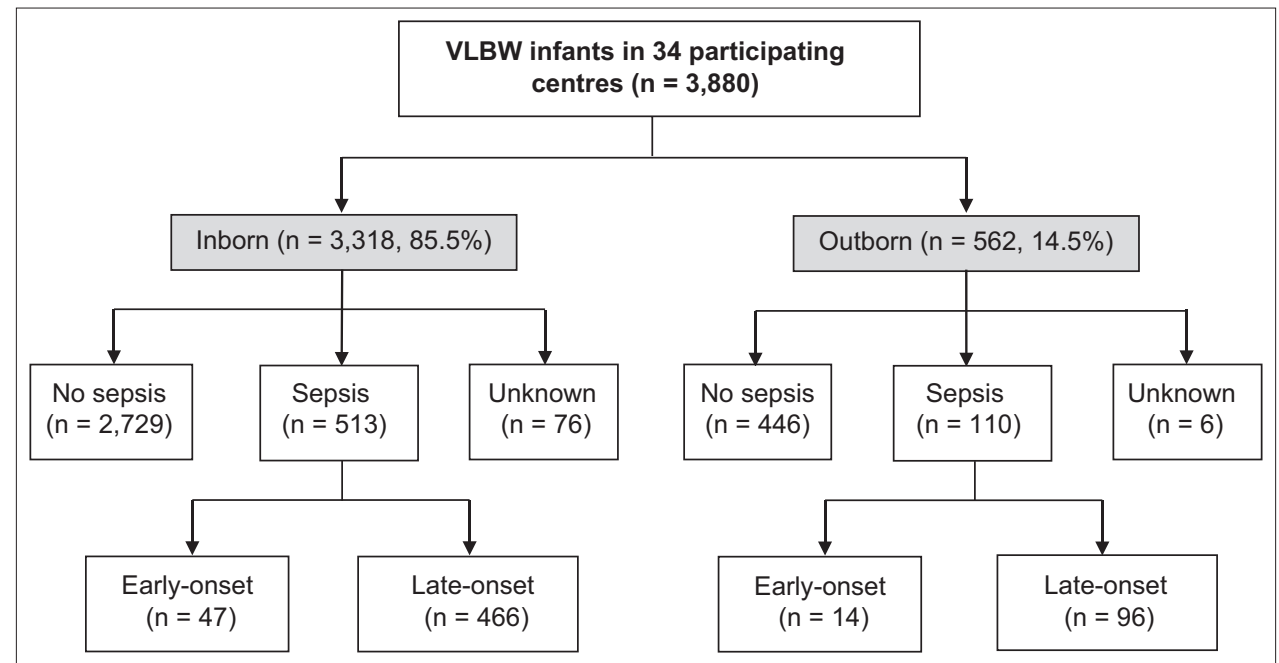

Fig. 1 STROBE diagram shows the details of very-low-birth-weight (VLBW) infants registered in the Malaysian National Neonatal Registry in 2010.
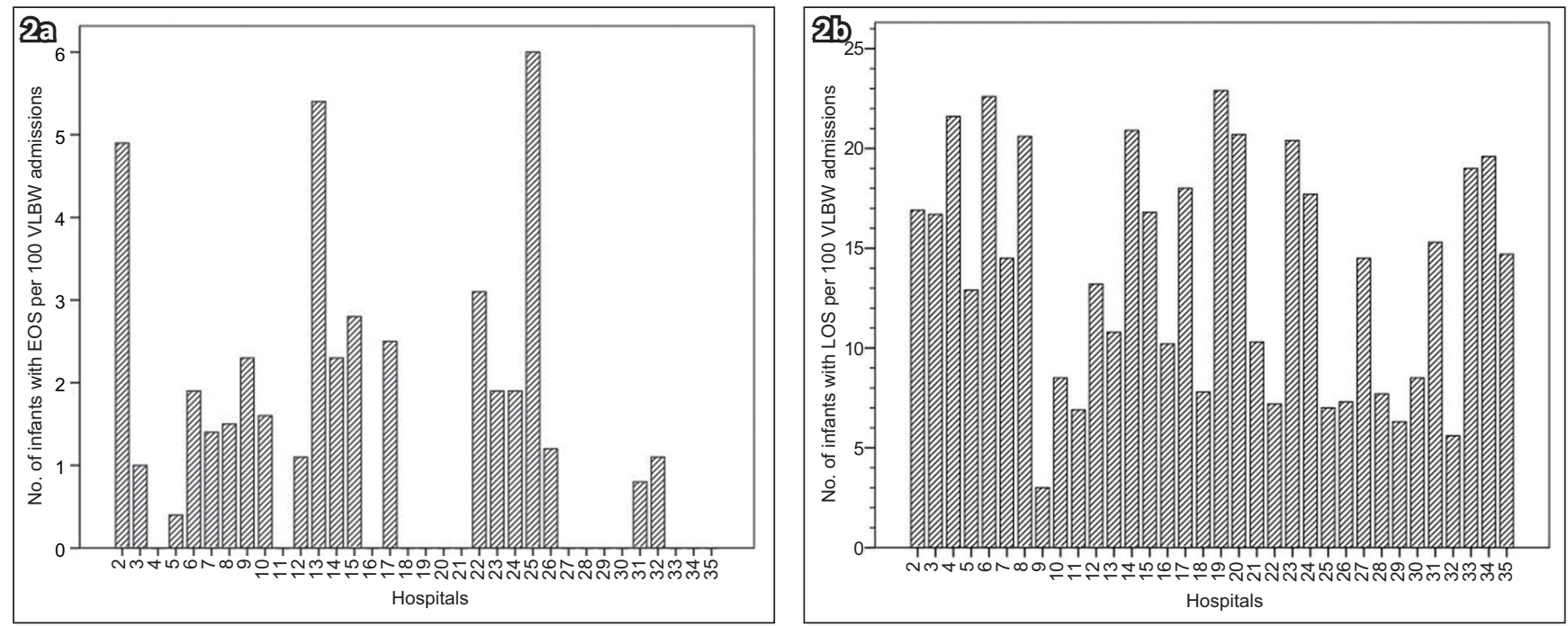

Fig. 2 Bar graphs show (a) early-onset sepsis (EOS) and (b) late-onset sepsis (LOS) rates of very-low-birth-weight (VLBW) infants in 34 neonatal intensive care units in 2010.

chest radiography findings. Necrotising enterocolitis (NEC) was diagnosed based on Stage II or III of Bell's criteria. ${ }^{(10)}$ Patent ductus arteriosus (PDA) was diagnosed based on echocardiographic evidence or the presence of a continuous heart murmur in the left second intercostal space. The central venous catheters used were umbilical, or percutaneously or surgically placed.

Statistical analysis was performed using PASW Statistics version 18.0 (SPSS Inc, Chicago, IL, USA). Descriptive statistics were reported as mean \pm standard deviation for continuous variables with a normal distribution, and median (interquartile range [IQR]) for those with a skewed distribution; categorical variables were reported as frequency and percentage. For comparison between groups in univariate analysis, chi-square test was used for categorical variables, Student's $t$-test for numerical variables with normal distribution and Mann-Whitney $U$ test for numerical variables with skewed distributions. For comparison of variables among three groups, the one-way analysis of variance (with Tukey and Games-Howell tests for post hoc analysis, where appropriate) and Kruskal-Wallis test were used for continuous variables, and chi-square test for categorical variables. Logistic regression analysis was performed to identify significant risk factors associated with EOS and LOS in VLBW infants between different groups. Potential risk factors used for logistic regression analysis were variables with $p$-value $<0.05$ identified in the univariate analysis. All tests were two-sided and p-value $<0.05$ indicated statistical significance.

\section{RESULTS}

The 34 NICUs received a total of 3,880 VLBW infants during the study period (Fig. 1). Sepsis was reported in 623 (16.1\%) infants: $61(9.8 \%)$ of these infants had EOS and $562(90.2 \%)$ had LOS. The sepsis status of 82 infants was unknown, including that of 67 infants who died in delivery rooms and six outborns who died shortly after admission.

Fig. 2 shows the EOS and LOS rates of infants in the 34 NICUs. The median EOS rate was 1.0\% (IQR 0\%, 2.0\%; range $0 \%-6.0 \%$ ). A total of $14 \mathrm{NICU}$ did not report any case of EOS, while $20 \mathrm{NICU}$ reported cases of EOS. The median LOS rate was $14.5 \%$ (IQR 7.8\%, 19.2\%; range 3.0\%-22.9\%). Eight NICUs had LOS rates below the first quartile (i.e. $<7.8 \%$, low group) with a 
Table I. Mortality rates of very-low-birth-weight infants and the causative organisms.

\begin{tabular}{|c|c|c|c|c|}
\hline \multirow[t]{2}{*}{ Organism } & \multicolumn{2}{|c|}{ Early-onset sepsis } & \multicolumn{2}{|c|}{ Late-onset sepsis } \\
\hline & Total $(n=61)$ & Mortality (n = 31) & Total $(n=562)$ & Mortality $(n=160)$ \\
\hline Gram-positive* & $31(50.8)$ & $11(35.5)$ & $264(47.0)$ & $53(20.1)$ \\
\hline Bacillus spp. & 0 & 0 & 10 & $3(30.0)$ \\
\hline Brevibacterium spp. & 0 & 0 & 1 & 0 \\
\hline Corynebacterium spp. & 1 & 0 & 1 & 0 \\
\hline Enterococcus spp. & 1 & 0 & 5 & $2(40.0)$ \\
\hline Listeria monocytogenes & 1 & $1(100.0)$ & 0 & 0 \\
\hline Coagulase-negative staphylococcus & 5 & 0 & 144 & $24(16.7)$ \\
\hline Methicillin-resistant Staphylococcus aureus & 3 & $2(66.7)$ & 56 & $17(30.4)$ \\
\hline S. aureus & 4 & $1(25.0)$ & 29 & $5(17.2)$ \\
\hline S. epidermidis & 0 & 0 & 3 & 0 \\
\hline Group B streptococcus & 16 & $7(43.8)$ & 6 & $2(33.3)$ \\
\hline Group D streptococcus & 0 & 0 & 5 & 0 \\
\hline Other Gram-positive bacteria & 0 & 0 & 4 & 0 \\
\hline Gram-negative & $30(49.2)$ & $20(66.7)$ & $298(53.0)$ & 107 (35.9) \\
\hline Acinetobacter spp. & 10 & $6(60.0)$ & 70 & $23(32.9)$ \\
\hline Burkholderia cepacia & 3 & $1(33.3)$ & 13 & $2(15.4)$ \\
\hline Citrobacter spp. & 0 & 0 & 2 & 0 \\
\hline Chryseobacterium spp. & 0 & 0 & 3 & $1(33.3)$ \\
\hline Enterobacter spp. & 2 & $1(50.0)$ & 9 & $7(77.8)$ \\
\hline Escherichia coli & 5 & $4(80.0)$ & 8 & $3(37.5)$ \\
\hline Klebsiella spp. & 2 & $2(100)$ & 83 & $33(39.8)$ \\
\hline Moraxella spp. & 0 & 0 & 1 & 0 \\
\hline Pseudomonas spp. & 4 & $3(75.0)$ & 49 & $22(44.9)$ \\
\hline Serratia marcescens & 0 & 0 & 16 & $1(6.3)$ \\
\hline Stenotrophomonas maltophilia & 0 & 0 & 10 & $6(60.0)$ \\
\hline Other Gram-negative bacteria & 4 & $3(75.0)$ & 5 & 0 \\
\hline Fungus & 0 & 0 & 29 & $9(31.0)$ \\
\hline
\end{tabular}

Data presented as no. (\%). Mortality percentages are calculated using the total number as denominator.

median rate of $7.0 \%$ (IQR 5.8\%, 7.3\%), $18 \mathrm{NICU}$ s had LOS rates between the first and third quartile (intermediate group) with a median LOS rate of $14.5 \%$ (IQR $10.3 \%, 16.8 \%$ ), and the remaining eight NICUs had rates above the third quartile (high group) with a median LOS rate of 20.8\% (IQR 20.5\%, 22.4\%). Both Grampositive and -negative organisms were equally common pathogens of EOS and LOS, with GBS (26.2\%) being the most common EOS pathogen and CoNS (25.6\%) the most common LOS pathogen (Table I). Overall mortality rates were high (191/623, 30.7\%).

Table II shows the maternal, perinatal and neonatal variables of all VLBW infants. Compared with infants without sepsis, EOS infants had significantly lower Apgar scores and gestational age, a higher likelihood of being outborn, more morbidities (RDS, pneumonia, NEC), treatment interventions (surfactant therapy, ventilation support, and total parenteral nutrition [TPN]) and types of resuscitation at birth, and a higher mortality rate. Logistic regression analysis showed that, when compared with infants without sepsis, the significant risk factors associated with EOS infants were gestational age (adjusted odds ratio [OR] 0.871, 95\% confidence interval $[\mathrm{Cl}] 0.790,0.961 ; \mathrm{p}=0.006)$, endotracheal tube (ETT) ventilation at birth (adjusted OR 2.651, 95\% Cl 1.367, 5.143; $p=0.004$ ) and pneumonia (adjusted OR 2.141, 95\% Cl 1.163 , 3.940; $p=0.014)$. Apgar scores, bag-and-mask ventilation and adrenaline therapy at birth, being outborn, surfactant therapy, NEC, conventional ventilation, high frequency oscillatory ventilation (HFOV), TPN and the use of a central venous catheter were not significant risk factors.

Compared with infants without sepsis (Table II), LOS infants had significantly higher rates of spontaneous vertex delivery (SVD), more resuscitation at birth, lower birth weight and gestational age, a higher likelihood of being AGA and outborn, lower admission temperatures, more clinical problems and treatment interventions, and a longer duration of hospital stay. Logistic regression analysis showed that, when compared with infants without sepsis, the significant risk factors associated with LOS infants were SVD (adjusted OR 1.414, 95\% Cl 1.163, 1.718; $\mathrm{p}=0.001$ ), birth weight (adjusted OR 0.999, 95\% Cl 0.999, $1.000 ; p=0.002$ ), pneumonia (adjusted OR 1.974, 95\% Cl 1.483, 2.330; $\mathrm{p}<0.0001$ ), PDA (adjusted OR 1.974, 95\% Cl 1.610, 2.419; $\mathrm{p}<0.0001$ ), NEC (adjusted OR 2.267, 95\% Cl 1.688, 3.044; $\mathrm{p}<0.0001$ ), continuous positive airway pressure (CPAP) therapy (adjusted OR 1.351, 95\% Cl 1.109, 1.647; $p=0.003$ ), conventional ventilation (adjusted OR 1.742, 95\% Cl 1.348, 2.251; $\mathrm{p}<0.0001$ ) and TPN (adjusted OR 2.413, 95\% Cl 1.904, 3.059; $p<0.0001)$. Oxygen therapy, resuscitation at birth with bag-and-mask or ETT ventilation, gestational age, growth status 
Table II. Comparison of maternal, perinatal and neonatal variables of very-low-birth-weight infants with and without sepsis.

\begin{tabular}{|c|c|c|c|c|c|}
\hline \multirow[t]{2}{*}{ Variable } & \multicolumn{5}{|c|}{ No. (\%) } \\
\hline & $\begin{array}{c}\text { Total } \\
(n=3,880)\end{array}$ & $\begin{array}{l}\text { No sepsis } \\
(n=3,175)\end{array}$ & $\begin{array}{c}\text { EOS } \\
(n=61)\end{array}$ & $\begin{array}{c}\text { LOS } \\
(n=562)\end{array}$ & $\begin{array}{l}\text { Unknown } \\
(n=82)\end{array}$ \\
\hline Age $(y r)^{*}$ & $\begin{array}{l}28(24,33) \\
(n=3,877)\end{array}$ & $\begin{array}{l}38(24,33) \\
(n=3,877)\end{array}$ & $\begin{array}{c}28(25,30) \\
(n=61)\end{array}$ & $\begin{array}{c}28(23,33) \\
(n=560)\end{array}$ & $\begin{array}{c}30(25,36) \\
(n=82)\end{array}$ \\
\hline Gravida* & $2(1,3)$ & $2(1,3)$ & $2(1,3)$ & $2(1,3)$ & $2(1,5)$ \\
\hline Parity* & $1(0,2)$ & $1(0,2)$ & $1(0,2)$ & $1(0,2)$ & $1(0,3)$ \\
\hline \multicolumn{6}{|l|}{ Ethnic group } \\
\hline Malay & $2,393(61.7)$ & $1,973(62.1)$ & $46(75.4)$ & 331 (58.9) & $43(52.4)$ \\
\hline Chinese & $349(9.0)$ & $283(8.9)$ & $4(6.6)$ & $56(10.0)$ & $6(7.3)$ \\
\hline Indian & $264(6.8)$ & $203(6.4)$ & $2(3.3)$ & $44(7.8)$ & $15(18.3)$ \\
\hline Sabahan & $218(5.6)$ & $179(5.6)$ & $1(1.6)$ & $28(5.0)$ & $10(12.2)$ \\
\hline Other & $100(2.6)$ & $83(2.6)$ & 0 & $13(2.3)$ & $4(4.9)$ \\
\hline Foreigner & $245(6.3)$ & $200(6.3)$ & $4(6.6)$ & $37(6.6)$ & $4(4.9)$ \\
\hline Previous abortion & $739(19.0)$ & $608(19.1)$ & $9(14.8)$ & $96(17.1)$ & $26(31.7)$ \\
\hline Diabetes mellitus & $485 / 3,697(13.1)$ & $396 / 3,030(13.1)$ & $9 / 59(15.3)$ & $62 / 532(11.7)$ & $18 / 76(23.7)$ \\
\hline Hypertension & $907 / 3,704(24.5)$ & $778 / 3,037(25.6)$ & $9 / 60(15.0)$ & $110 / 531(20.7)$ & $10 / 76(13.2)$ \\
\hline Chorioamnionitis & $166 / 3,692(4.5)$ & $131 / 3,027(4.3)$ & $4 / 59(6.8)$ & $26 / 531(4.9)$ & $5 / 75(6.7)$ \\
\hline Intrapartum antibiotics & $726 / 3,737(19.4)$ & $576 / 3,061(18.8)$ & $13 / 59(22.0)$ & $126 / 536(23.5)$ & $11 / 81(13.6)$ \\
\hline Antenatal steroid & $2,419 / 3,736(64.7)$ & $1,984 / 3,057(64.9)$ & $31 / 60(51.7)$ & $381 / 540(70.6)$ & $23 / 79(29.1)$ \\
\hline \multicolumn{6}{|l|}{ Mode of delivery } \\
\hline$S V D$ & $2,019(52.0)$ & $1,578(49.7)$ & $41(67.2)$ & $327(58.2)^{\S}$ & $73(89.0)$ \\
\hline Instrumental & $10(0.3)$ & $9(0.3)$ & 0 & $1(0.2)$ & 0 \\
\hline LSCS & $1,850(47.7)$ & $1,587(50.0)$ & $20(32.8)$ & $234(41.6)$ & $9(11.0)$ \\
\hline \multicolumn{6}{|l|}{ Infant } \\
\hline Female & $1,783(46.0)$ & $1,448(45.6)$ & $22(36.1)$ & $270(48.0)$ & $43(52.4)$ \\
\hline Outborn & $562(14.5)$ & $446(14.0)$ & $14(23.0)^{\neq}$ & $96(17.1)^{*}$ & $6(7.3)$ \\
\hline Singleton & $3,304(85.2)$ & $2,685(84.6)$ & $54(88.5)$ & $497(88.4)$ & $68(82.9)$ \\
\hline \multicolumn{6}{|l|}{ Apgar score* } \\
\hline At $1 \mathrm{~min}$ & $\begin{array}{c}7(5,9) \\
(n=3,669)\end{array}$ & $\begin{array}{c}7(5,9) \\
(n=3,005)\end{array}$ & $\begin{array}{l}6(3,8)^{*} \\
(n=56)\end{array}$ & $\begin{array}{c}7(5,8) \\
(n=536)\end{array}$ & $\begin{array}{l}2(1,3) \\
(n=72)\end{array}$ \\
\hline At $5 \mathrm{~min}$ & $\begin{array}{c}9(7,9) \\
(n=3,424)\end{array}$ & $\begin{array}{c}9(5,9) \\
(n=2,818)\end{array}$ & $\begin{array}{c}8(5.8,9.0)^{\S} \\
(n=54)\end{array}$ & $\begin{array}{c}9(7,9) \\
(n=493)\end{array}$ & $\begin{array}{l}1(1,3) \\
(n=59)\end{array}$ \\
\hline \multicolumn{6}{|l|}{ Resuscitation at birth } \\
\hline Oxygen & $3,112(80.2)$ & $2,540(80.0)$ & $53(86.9)$ & $489(87.0)^{\pi}$ & $30(36.6)$ \\
\hline Bag-and-mask ventilation & $2,218(57.2)$ & $1,775(55.9)$ & $46(75.4)^{\S}$ & $384(68.3)^{\pi}$ & $13(15.9)$ \\
\hline Chest compression & $149(3.8)$ & $121(3.8)$ & $5(8.2)$ & $14(2.5)$ & $9(11.0)$ \\
\hline ETT ventilation & $2,065(53.2)$ & $1,625(51.2)$ & $47(77.0)^{9}$ & $383(68.1)^{q}$ & $10(12.2)$ \\
\hline Adrenaline & $101(2.6)$ & $81(2.6)$ & $5(8.2)^{\ddagger}$ & $7(1.2)$ & $8(9.8)$ \\
\hline Birth weight* (g) & $1,160(930,1,350)$ & $1,190(960,1,370)$ & $1,150(925,1,335)$ & $1,055(874,1,250)^{\natural}$ & $611(554,898)$ \\
\hline Gestation $^{\dagger}$ (wk) & $29.5 \pm 3.1$ & $29.8 \pm 3.1$ & $28.4 \pm 2.6$ & $28.1 \pm 2.6^{\natural}$ & $26.3 \pm 3.7$ \\
\hline \multicolumn{6}{|l|}{ Intrauterine growth status } \\
\hline$A G A$ & $2,366(61.0)$ & $1,894(59.7)$ & $38(62.3)$ & $383(68.1)^{\S}$ & $51(62.2)$ \\
\hline$L G A$ & $82(2.1)$ & $68(2.1)$ & $1(1.6)$ & $12(2.1)$ & $1(1.2)$ \\
\hline$S G A$ & $1,432(36.9)$ & $1,213(38.2)$ & $22(36.1)$ & $167(29.7)$ & $30(36.6)$ \\
\hline Admission temperature ${ }^{*}\left({ }^{\circ} \mathrm{C}\right)$ & $36.0(35.2,36.5)$ & $36.5(36.0,36.8)$ & $36.0(35.2,36.5)$ & $36.0(35.0,36.5)^{\pi}$ & $\begin{array}{c}35.5(35.0,36.0) \\
(n=25)\end{array}$ \\
\hline RDS & $2,844 / 3,798(74.9)$ & $2,298(72.4)$ & $54(88.5)^{\S}$ & $492(87.5)^{9}$ & - \\
\hline Surfactant therapy & $2,259 / 3,798$ (59.5) & $1,792(56.4)$ & $49(80.3)^{9}$ & $418(74.4)^{\natural}$ & 0 \\
\hline
\end{tabular}




\begin{tabular}{|c|c|c|c|c|c|}
\hline \multirow[t]{2}{*}{ Variable } & \multicolumn{5}{|c|}{ No. (\%) } \\
\hline & $\begin{array}{c}\text { Total } \\
(n=3,880)\end{array}$ & $\begin{array}{l}\text { No sepsis } \\
(n=3,175)\end{array}$ & $\begin{array}{c}\text { EOS } \\
(n=61)\end{array}$ & $\begin{array}{c}\text { LOS } \\
(n=562)\end{array}$ & $\begin{array}{l}\text { Unknown } \\
(n=82)\end{array}$ \\
\hline NEC & $262(6.8)$ & $162(5.1)$ & $7(11.5)^{*}$ & $93(16.5)^{9}$ & 0 \\
\hline Major congenital anomalies & $295(7.6)$ & $235(7.4)$ & $5(8.2)$ & $55(9.8)$ & 0 \\
\hline CPAP & $1,653(42.6)$ & $1,331(41.9)$ & $24(39.3)$ & $298(53.0)^{\natural}$ & 0 \\
\hline Conventional ventilation & $2,501(64.5)$ & $1,981(62.4)$ & $50(82.0)^{\S}$ & $470(83.6)^{9}$ & 0 \\
\hline HFOV & $261(6.7)$ & $195(6.1)$ & $11(18.0)^{\S}$ & $55(9.8)^{\S}$ & 0 \\
\hline TPN & $1,961(50.5)$ & $1,480(46.6)$ & $41(67.2)^{\S}$ & $440(78.3)^{\natural}$ & 0 \\
\hline CVC use & $2,322(59.8)$ & $1,835(57.8)$ & $47(77.0)^{\ddagger}$ & $440(78.3)^{n}$ & 0 \\
\hline Death & $1,037(26.7)$ & $846(26.6)$ & $31(50.8)^{n}$ & $160(28.5)$ & $82(100.0)$ \\
\hline \multicolumn{6}{|l|}{ Duration of hospital stay* (day) } \\
\hline Total & $35(15,56)$ & $34(15,53)$ & $27(3,56)$ & $54(32,79)^{n}$ & 0 \\
\hline Survivors & $\begin{array}{r}44.0(31.0,63.0) \\
(n=2,843)\end{array}$ & $\begin{array}{c}42.0(30.0,59.0) \\
(n=2,411)\end{array}$ & $\begin{array}{c}49.0(32.0,73.5) \\
(n=30)\end{array}$ & $\begin{array}{c}61.5(45.0,86.0)^{n} \\
(n=402)\end{array}$ & - \\
\hline
\end{tabular}

Value of $\mathrm{n}$ provided for variables with missing data. *Data presented as median (interquartile range). ${ }^{\text {DData }}$ presented as mean \pm standard deviation. Statistically significant at $\neq p<0.05, \S_{p}<0.001,{ }^{\uparrow} p<0.0001$ compared to infants without sepsis. AGA: appropriate for gestational age; CPAP: continuous positive airway pressure; CVC: central venous catheter; ETT: endotracheal tube; HFOV: high frequency oscillatory ventilation; LGA: large for gestational age; LSCS: lower segment Caesarean section; NEC: necrotising enterocolitis; PDA: patent ductus arteriosus; RDS: respiratory distress syndrome; SGA: small for gestational age; SVD: spontaneous vertex delivery; TPN: total parenteral nutrition

Table III. Comparison of patient loads of Malaysian neonatal intensive care units (NICUs) with and without early-onset sepsis (EOS) in infants with very low birth weight (VLBW) and extremely low birth weight (ELBW).

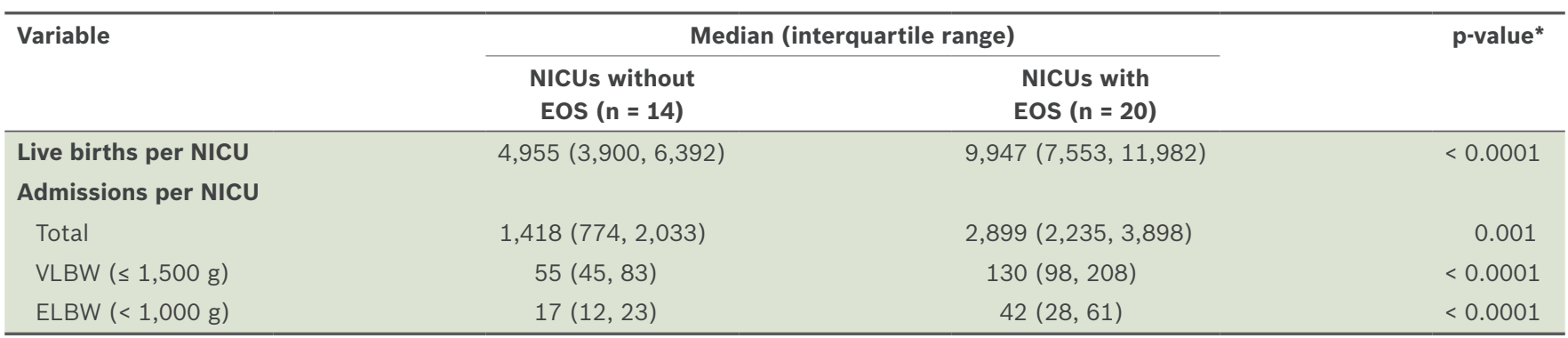

* Statistically significant at $\mathrm{p}<0.05$.

at birth, being outborn, admission temperature, RDS, surfactant therapy, HFOV and use of a central venous catheter were not significant risk factors.

There were no significant differences in maternal, intrapartum and neonatal variables (Table II) between EOS and LOS infants, except that EOS infants had significantly lower Apgar scores at one minute $(p=0.037)$ and five minutes $(p=0.023)$ of life, more chest compression $(p=0.030)$ and adrenaline therapy $(p=0.004)$ at birth, and a higher mortality rate $(p<0.0001)$. LOS infants had a significantly higher rate of exposure to antenatal steroids $(p=0.007)$, lower admission temperature $(p=0.013)$, more PDA $(p=0.019)$ and CPAP therapy $(p=0.042)$, less HFOV $(p=0.047)$, and a longer duration of hospital stay $(p<0.0001)$ among the survivors $(p=0.030)$.

Tables III and IV show a comparison of the patient loads and characteristics of VLBW infants in NICUs with and without EOS. Compared with NICUs without EOS infants, those with EOS infants had significantly more live births, admissions, VLBW and extremely-low-birth-weight (ELBW) infants, and outborns. Furthermore, in these NICUs, a significantly higher proportion of mothers of VLBW infants had a history of abortion and use of antenatal steroids or intrapartum antibiotics; the infants had lower birth weight and gestational age; there were also more infants requiring bag-and-mask ventilation, chest compression, ETT ventilation and adrenaline at birth, as well as higher incidences of surfactant therapy, pneumonia and insertion of central venous catheters.

Tables $\mathrm{V}$ and $\mathrm{VI}$ show a comparison of the patient loads and variables of NICUs with low, intermediate and high LOS rates. There was no significant difference in total, VLBW and ELBW admissions to the NICU among these three groups. When compared with VLBW infants in NICUs with low LOS rates, those in NICUs with high LOS rates had significantly younger mothers ( $p<0.001)$, higher birth weight $(p<0.001)$ and gestational age $(p=0.027)$, a higher likelihood of being AGA $(p<0.0001)$, outborn ( $p=0.033$ ) and singleton $(p=0.015)$, lower Apgar score at one minute of life $(p<0.001)$, and were more likely to require oxygen therapy $(p=0.002)$, bag-and-mask ventilation $(p<0.001)$ and ETT ventilation $(p=0.011)$ at birth. Such infants also had lower admission temperature $(p=0.017)$, more RDS $(p<0.001)$, surfactant therapy $(p<0.001)$, pneumonia $(p<0.001)$ and PDA $(p<0.001)$, used less intrapartum antibiotics $(p=0.038)$, and received more conventional mechanical ventilation $(p=0.034)$ and TPN $(p<0.001)$. 
Table IV. Comparison of maternal, perinatal and neonatal variables in very-low-birth-weight infants in Malaysian neonatal intensive care units (NICUs) with and without early-onset sepsis (EOS).

\begin{tabular}{|c|c|c|c|}
\hline \multirow[t]{2}{*}{ Variable } & \multicolumn{2}{|c|}{ No. (\%) } & \multirow[t]{2}{*}{ p-value } \\
\hline & $\begin{array}{l}\text { NICUs without } \\
\text { EOS }(n=970)\end{array}$ & $\begin{array}{c}\text { NICUs with } \\
\text { EOS }(n=2,910)\end{array}$ & \\
\hline \multicolumn{4}{|l|}{ Maternal } \\
\hline Age $^{*}(y r)$ & $28(23,33)$ & $28(24,33)$ & 0.073 \\
\hline Gravida* & $2(1,4)$ & $2(1,3)$ & 0.238 \\
\hline Parity* & $1(0,2)$ & $1(0,2)$ & 0.659 \\
\hline Previous abortion & $163(16.8)$ & $576(19.8)$ & $0.040^{\ddagger}$ \\
\hline Diabetes mellitus & $128 / 914(14.0)$ & $357 / 2,783(12.8)$ & 0.361 \\
\hline Hypertension & $227 / 915(24.8)$ & $680 / 2,789(24.4)$ & 0.794 \\
\hline Chorioamnionitis & $50 / 917(5.5)$ & $116 / 2,775(4.2)$ & 0.107 \\
\hline Antenatal steroid & $592 / 955(62.0)$ & $1,827 / 2,781(65.7)$ & $0.039^{*}$ \\
\hline Intrapartum antibiotics & $162 / 944(17.2)$ & $564 / 2,793(20.2)$ & $0.042^{\ddagger}$ \\
\hline \multicolumn{4}{|l|}{ Infant } \\
\hline Female & $438(45.2)$ & $1,345(46.2)$ & 0.831 \\
\hline Outborn & 115 (11.9) & $447(15.4)$ & $0.007^{*}$ \\
\hline Singleton & $838(86.4)$ & $2,466(84.7)$ & 0.211 \\
\hline Birth weight* (g) & $1,180(940,1,459)$ & $1,150(925,1,350)$ & $0.039^{*}$ \\
\hline Gestation* (wk) & $30(27,32)$ & $29(27,31)$ & $0.021^{*}$ \\
\hline Intrauterine growth status & & & 0.579 \\
\hline$A G A$ & $605(62.4)$ & $1,761(60.5)$ & \\
\hline$L G A$ & $19(2.0)$ & $63(2.2)$ & \\
\hline$S G A$ & $346(35.7)$ & $1,086(37.3)$ & \\
\hline LSCS delivery & $451(46.5)$ & $1,399(48.1)$ & 0.742 \\
\hline \multicolumn{4}{|l|}{ Apgar score* } \\
\hline At $1 \mathrm{~min}$ & $\begin{array}{l}7(5,9) \\
(n=922)\end{array}$ & $\begin{array}{c}7(5,9) \\
(n=2,747)\end{array}$ & 0.194 \\
\hline At $5 \mathrm{~min}$ & $\begin{array}{c}9(8,9) \\
(n=856)\end{array}$ & $\begin{array}{c}9(7,9) \\
(n=2,568)\end{array}$ & 0.667 \\
\hline \multicolumn{4}{|l|}{ Resuscitation at birth } \\
\hline Oxygen & 785 (80.9) & $2,327(80.0)$ & 0.515 \\
\hline Bag-and-mask ventilation & $498(51.3)$ & $1,720(59.1)$ & $<0.0001^{*}$ \\
\hline Chest compression & $20(2.1)$ & $129(4.4)$ & $0.001^{*}$ \\
\hline ETT ventilation & $468(48.2)$ & $1,597(54.9)$ & $<0.0001^{*}$ \\
\hline Adrenaline & $11(1.1)$ & $90(3.1)$ & $0.001^{*}$ \\
\hline Admission temperature ${ }^{\dagger}\left({ }^{\circ} \mathrm{C}\right)$ & $\begin{array}{c}35.80 \pm 0.98 \\
(n=959)\end{array}$ & $\begin{array}{c}35.80 \pm 1.06 \\
(n=2,864)\end{array}$ & 0.072 \\
\hline Respiratory distress syndrome & $710 / 955(74.3)$ & $2,134 / 2,843(75.1)$ & 0.659 \\
\hline Surfactant therapy & $541 / 955(56.6)$ & $1,718 / 2,843(60.4)$ & $0.04^{\ddagger}$ \\
\hline Pneumonia & 115 (11.9) & $515(17.7)$ & $<0.0001^{*}$ \\
\hline Central venous catheter use & $533 / 951(56.0)$ & $1,789 / 2,813(63.6)$ & $<0.0001^{*}$ \\
\hline
\end{tabular}

Value of $\mathrm{n}$ provided for variables with missing data. *Data presented as median (interquartile range). ${ }^{\dagger}$ Data presented as mean \pm standard deviation. ${ }^{*}$ Statistically significant at $p<0.05$. AGA: appropriate for gestational age; ETT: endotracheal tube; LGA: large for gestational age; LSCS: lower segment Caesarean section; SGA: small for gestational age

Table V. Comparison of patient loads of Malaysian neonatal intensive care units (NICUs) with differing late-onset sepsis (LOS) rates in very-low-birth-weight (VLBW) infants.

\begin{tabular}{|c|c|c|c|c|}
\hline \multirow[t]{2}{*}{ Variable } & \multicolumn{3}{|c|}{ Median (interquartile range) } & \multirow[t]{2}{*}{ p-value } \\
\hline & Low $\operatorname{LOS}^{*}(n=8)$ & Intermediate $\operatorname{LOS}^{+}(n=18)$ & High LOS* $(n=8)$ & \\
\hline Live births per NICU & $8,826(4,752,10,209)$ & $7,592(5,026,10,424)$ & $5,853(4,875,12,221)$ & 0.931 \\
\hline \multicolumn{5}{|l|}{ Admissions per NICU } \\
\hline Total & $2,945(2,247,4,566)$ & $2,065(1,494,3,190)$ & $1,369(931,4,461)$ & 0.310 \\
\hline VLBW $(\leq 1,500 \mathrm{~g})$ & $97(52,124)$ & $113(69,208)$ & $95(51,197)$ & 0.655 \\
\hline
\end{tabular}

LOS rate $*<7.8 \%, t_{\geq} 7.8 \%$ and $<19.2 \%, \neq \geq 19.2 \%$. ELBW: extremely low birth weight 
Table VI. Comparison of maternal, perinatal and neonatal variables in Malaysian neonatal intensive care units with differing late-onset sepsis (LOS) rates in very-low-birth-weight infants.

\begin{tabular}{|c|c|c|c|c|}
\hline \multirow[t]{2}{*}{ Variable } & \multicolumn{3}{|c|}{ No. (\%) } & \multirow[t]{2}{*}{ p-value } \\
\hline & Low LOS* $(n=746)$ & Intermediate $\operatorname{LOS}^{+}(n=2,216)$ & High LOS* $(n=918)$ & \\
\hline \multicolumn{5}{|l|}{ Maternal } \\
\hline $\operatorname{Age}^{\S}(y r)$ & $29.3 \pm 6.5$ & $28.7 \pm 6.5$ & $27.9 \pm 7.1$ & $<0.001^{\|}$ \\
\hline Gravida" & $2(1,4)$ & $2(1,3)$ & $2(1,4)$ & 0.075 \\
\hline Parity" & $1(0,2)$ & $1(0,2)$ & $1(0,2)$ & 0.063 \\
\hline Hypertension & $159 / 716(22.2)$ & $532 / 2,107(25.2)$ & $216 / 881(24.5)$ & 0.262 \\
\hline Chorioamnionitis & $28 / 717(3.9)$ & $104 / 2,095(5.0)$ & $34 / 880$ (3.9) & 0.291 \\
\hline Antenatal steroid & $447 / 726(61.6)$ & $1,380 / 2,107(65.5)$ & $592 / 903(65.6)$ & 0.136 \\
\hline Intrapartum antibiotics & $169 / 724(23.3)$ & $385 / 2,114(18.2)$ & $172 / 899(19.1)$ & $0.010^{\|}$ \\
\hline \multicolumn{5}{|l|}{ Infant } \\
\hline Female & $343(46.0)$ & $1,011(45.6)$ & $429(46.7)$ & 0.977 \\
\hline Outborn & $81(10.9)$ & $349(15.7)$ & $132(14.4)$ & $0.00{ }^{\|}$ \\
\hline Singleton & $621(83.2)$ & $1,880(84.8)$ & $803(87.5)$ & $0.044^{\|}$ \\
\hline Birth weight ${ }^{\S}(\mathrm{g})$ & $1,102 \pm 274$ & $1,119 \pm 267$ & $1,148 \pm 266$ & $0.001^{\|}$ \\
\hline Gestation $^{\S}$ (wk) & $29.4 \pm 3.1$ & $29.5 \pm 3.1$ & $29.8 \pm 3.1$ & $0.028^{\|}$ \\
\hline \multicolumn{5}{|l|}{ Intrauterine growth status } \\
\hline$A G A$ & $395(52.9)$ & $1,358(61.3)$ & $613(66.8)$ & $<0.001^{\|}$ \\
\hline$L G A$ & $16(2.1)$ & $41(1.9)$ & $25(2.7)$ & 0.055 \\
\hline$S G A$ & $335(44.9)$ & $817(36.9)$ & $280(30.5)$ & Reference \\
\hline LSCS delivery & $346(46.4)$ & $1,099(49.6)$ & $405(44.1)$ & 0.100 \\
\hline \multicolumn{5}{|l|}{ Apgar score" } \\
\hline At $1 \mathrm{~min}$ & $\begin{array}{c}8(5,9) \\
(n=715)\end{array}$ & $\begin{array}{c}7(5,8) \\
(n=2,082)\end{array}$ & $\begin{array}{c}7(5,8) \\
(n=872)\end{array}$ & $<0.001^{\|}$ \\
\hline At $5 \mathrm{~min}$ & $\begin{array}{c}9(7,9) \\
(n=662)\end{array}$ & $\begin{array}{c}9(7,9) \\
(n=1,967)\end{array}$ & $\begin{array}{c}9(8,9) \\
(n=795)\end{array}$ & 0.102 \\
\hline \multicolumn{5}{|l|}{ Resuscitation at birth } \\
\hline Oxygen & $575(77.1)$ & $1,775(80.1)$ & $762(83.0)$ & $0.010^{\|}$ \\
\hline Bag-and-mask ventilation & $379(50.8)$ & $1,289(58.2)$ & $550(59.9)$ & $<0.001^{\|}$ \\
\hline Chest compression & $21(2.8)$ & $84(3.8)$ & $44(4.8)$ & 0.11 \\
\hline $\begin{array}{l}\text { Endotracheal tube } \\
\text { ventilation }\end{array}$ & $370(49.6)$ & $1,182(53.3)$ & $513(55.9)$ & $0.038^{\|}$ \\
\hline Adrenaline & $14(1.9)$ & $66(3.0)$ & $21(2.3)$ & 0.208 \\
\hline Admission temperature $\left.{ }^{\natural}{ }^{\circ} \mathrm{C}\right)$ & $\begin{array}{c}36.2(35.9,36.5) \\
(n=731)\end{array}$ & $\begin{array}{c}36.0(35.0,36.5) \\
(n=2,177)\end{array}$ & $\begin{array}{c}36.1(35.3,36.5) \\
(n=915)\end{array}$ & $<0.001^{\|}$ \\
\hline RDS & $527 / 722(73.0)$ & $1,596 / 2,165(73.7)$ & $721 / 911(79.1)$ & $0.003^{\|}$ \\
\hline Surfactant therapy & $380 / 722(52.6)$ & $1,287 / 2,165(59.4)$ & $592 / 911(65.0)$ & $<0.001^{\|}$ \\
\hline Pneumonia & $67(9.0)$ & $425(19.2)$ & $138(15.0)$ & $<0.001^{\|}$ \\
\hline PDA & $158 / 722(21.9)$ & $498 / 2,165(23.0)$ & $275 / 911(30.2)$ & $<0.001^{\|}$ \\
\hline Necrotising enterocolitis & $40 / 722(5.5)$ & $172 / 2,165(7.9)$ & $50 / 911(5.5)$ & $0.014^{\|}$ \\
\hline CPAP & $254 / 746(34.0)$ & $1,053 / 2,216(47.5)$ & $346 / 918(37.7)$ & $<0.001^{\|}$ \\
\hline Conventional ventilation & $481(64.5)$ & $1,383(62.4)$ & $637(69.4)$ & $0.001^{\Perp}$ \\
\hline HFOV & $63(8.4)$ & $141(6.4)$ & $57(6.2)$ & 0.112 \\
\hline Total parenteral nutrition & $335 / 722(46.4)$ & $1,096 / 2,165(50.6)$ & $530 / 911(58.2)$ & $<0.001^{\|}$ \\
\hline Central venous catheter use & $460 / 715(64.3)$ & $1,288 / 2,141(60.2)$ & $574 / 908$ (63.2) & 0.077 \\
\hline
\end{tabular}

LOS rate $*<7.8 \%, \uparrow \geq 7.8$ and $<19.2, \ddagger \geq 19.2 \%$. §Data presented as mean \pm standard deviation. "Data presented as median (interquartile range). IIStatistically significant $p<0.05$. AGA: appropriate for gestational age; CPAP: continuous positive airway pressure; HFOV: high frequency oscillatory ventilation; LGA: large for gestational age; LSCS: lower segment Caesarean section; PDA: patent ductus arteriosus; RDS: respiratory distress syndrome; SGA: small for gestational age

\section{DISCUSSION}

This study showed that EOS was not common in Malaysian VLBW infants, but that it had a high mortality rate. The EOS and LOS rates of Malaysian NICUs were comparable with those of some networks/registries, ${ }^{(3,11)}$ but lower than the rates reported by others. ${ }^{(12,13)}$

Unlike reports in which Gram-negative organisms were the predominant pathogens ${ }^{(3,11,14-16)}$ both Gram-positive 
and -negative organisms were common EOS pathogens in Malaysian VLBW infants. GBS, the most common pathogen of EOS in our NICUs, produced a higher mortality rate than in other reports. ${ }^{(11,17)}$ Multivariate analysis showed that lower gestational age, resuscitation at birth with ETT ventilation and pneumonia were the only significant risk factors associated with EOS among VLBW infants, compared to those without EOS. Stoll et al have proposed that ascending amniotic infections by maternal vaginal flora are responsible for most cases of EOS. ${ }^{(15)}$ In the present study, we suspect that chorioamnionitis was underreported, as almost a quarter of EOS infants received intrapartum antibiotics. ${ }^{(18)}$ Intrapartum antibiotics have been reported to be effective in the prevention and treatment of chorioamnionitis. ${ }^{(15)}$ Similar to Klinger et al, ${ }^{(18)}$ we found that delivery room ETT ventilation was a significant risk factor for EOS. Compromise of respiratory mucosa due to endotracheal intubation and contamination of the ETT during resuscitation were possible underlying mechanisms of EOS and pneumonia.

Some networks have found that Gram-positive organisms were the most common pathogens of LOS. ${ }^{(1-3,11,12,16,19,20)}$ However, in the current study, both Gram-positive and -negative organisms were found to be common pathogens of LOS, with CoNS being the most common Gram-positive pathogen in Malaysian NICUs. Unlike NICUs in other countries, ${ }^{(1,11,19-21)}$ Escherichia coli was not found to be the most common Gram-negative LOS pathogen in Malaysian NICUs in our study. Fungal infection was also less common $(4.7 \%)$ than in other networks/registries, which reported an incidence of more than $10.0 \% .^{(1,11,12,19,20,22)}$ In our study, multivariate analysis showed that, when compared with VLBW infants without LOS, the significant risk factors associated with VLBW infants with LOS were lower birth weight, SVD, pneumonia, PDA, NEC, CPAP therapy, conventional ventilation and TPN.

This study also showed that there were differences between EOS and LOS infants. EOS infants had significantly lower Apgar scores, hence requiring more extensive resuscitation procedures at birth than LOS infants. However, LOS infants had significantly higher rates of exposure to antenatal steroids, lower admission temperature, more PDA and higher rates of respiratory support. A possible mechanism underlying the increased risk of LOS is the suppression of the immature immune system by antenatal steroids. Generally, infants with hypothermia and PDA necessitated more intervention and a longer duration of respiratory support, which could subsequently contribute to sepsis.

Similar to the findings in other networks, ${ }^{(1,5-7)}$ we also observed a wide variability in sepsis rates. When compared with Malaysian NICUs that reported no EOS in their VLBW infants, those with reported EOS in their VLBW infants had significantly higher patient loads (number of live births, admissions, VLBW and ELBW infants, outborn infants) and more therapeutic interventions at birth (resuscitation procedures, surfactant therapy and insertion of CVCs). Furthermore, they had a significantly larger number of mothers with a previous history of abortions and use of intrapartum antibiotics, suggesting that intrapartum infections were more common among populations giving birth to VLBW infants served by these NICUs. Unfortunately, data on prolonged rupture of membranes and maternal intrapartum fever were not available in the MNNR database. When compared with NICUs with low LOS rates, those with high LOS rates had bigger, more mature infants, more outborn infants, and used less intrapartum antibiotics. These NICUs also had significantly more sick infants (lower one-minute Apgar scores, more resuscitation at birth, lower admission temperature, more RDS, pneumonia and PDA) who required more ventilator support and TPN, which increased their risk of LOS.

A literature review showed that no studies have reported on the association of these risk factors with inter-institutional variability. Our findings suggest that when comparing outcomes of infection control strategies among different NICUs, the factors listed are potential confounders. Therefore, the ability to cope with higher patient loads and improvement of standards of perinatal care are issues that need to be addressed concomitantly when undertaking evidence-based preventive strategies to reduce sepsis rates in Malaysian NICUs.

The limitations of this study include: possible underreporting of LOS, as only the first episode of sepsis was entered in the database; lack of data on the incidence of prolonged rupture of membranes and maternal intrapartum fever; and lack of data on the duration of CVC use, which could be a significant factor associated with inter-institutional variations in sepsis rates.

In conclusion, the current study found that patient loads, patient characteristics and perinatal interventions were significant factors associated with inter-institutional variations of sepsis rates in Malaysian NICUs.

\section{ACKNOWLEDGEMENTS}

We would like to thank the Director General of the Ministry of Health, Malaysia, for giving us permission to publish this paper. The following were members of the 2009/2010 Steering Committee of the MNNR: Irene Guat-Sim Cheah, Jimmy KokFoo Lee, Thian-Lian Soo, Seok-Chiong Chee, Noraida Ramli, Zuraidah Abdul Latif, Siew-Hong Neoh, Meow-Keong Thong and Nem-Yun Boo. The following were site coordinators of participating hospitals: Zuraidah Abdul Latif (Hospital Ampang), Zainah Shaik Hedra (Hospital Batu Pahat), Ananda Mohan Lal (Hospital Bintulu), Baizura Jamaluddin (Hospital Kajang), Song-Hai Lim (Hospital Keningau), Siew-Hong Neoh (Hospital Kuala Lumpur), Anusha Palakrishanan (Hospital Kuala Pilah), Poy-Lee Leow (Hospital Melaka), Siao-Hean Teh (Miri Hospital), Revathy Nallusamy (Pulau Pinang Hospital), Nor Diana Yahya (Putrajaya Hospital), Noor Khatijah Nurani (Hospital Raja Permaisuri Bainun), Hasmawati Hassan (Hospital Raja Perempuan Zainab II), Maneet Kaur (Sabah Women and Children Hospital), Lee-Gaik Chan (Sarawak General Hospital), Angeline Yeoh (Seberang Jaya Hospital), Rohaizah Borhan (Serdang Hospital), Seok-Chiong Chee (Selayang Hospital), Sow-Keng Chan (Seri Manjung Hospital), Audrey Chae-Hee Chieng (Sibu Hospital), Thiyagar Nadarajah (Hospital Sultan Abdul Halim), Keng-Hwang Teh (Hospital Sultanah Bahiyah), Neesa Amran (Hospital Sultan Haji Ahmad Shah), Unnikrishnan Gopinathan (Sultanah Aminah 
Hospital), Pui-Ying Tam (Sultanah Fatimah Hospital), Sharifah Huda Engku Alwi (Sultanah Nur Zahirah Hospital), Kwee-Ching See (Sungai Buloh Hospital), Saiful Rijal (Taiping Hospital), Nizam Malik Bali Mohamed (Teluk Intan Hospital), Choy-Nyok Chin (Tengku Ampuan Afzan Hospital), Yogeswary Sithamparanathan (Tengku Ampuan Rahimah Hospital), Hazimah Mohd Yusoff (Tuanku Fauziah Hospital), Umathevi Paramasivam (Tuanku Ja'afar Hospital), Noraida Ramli (Universiti Sains Malaysia Hospital).

\section{REFERENCES}

1. Stoll BJ, Gordon T, Korones SB, et al. Late-onset sepsis in very low birth weight neonates: a report from the National Institute of Child Health and Human Development Neonatal Research Network. J Pediatr 1996; 129:63-71.

2. Fanaroff AA, Korones SB, Wright LL, et al. Incidence, presenting features, risk factors and significance of late onset septicemia in very low birth weight infants. The National Institute of Child Health and Human Development Neonatal Research Network. Pediatr Infect Dis J 1998; 17:593-8.

3. Stoll BJ, Hansen N. Infections in VLBW infants: studies from the NICHD Neonatal Research Network. Semin Perinatol 2003; 27:293-301.

4. Tröger B, Göpel W, Faust K, et al; German Neonatal Network. Risk for late-onset blood-culture proven sepsis in very-low-birth weight infants born small for gestational age: a large multicenter study from the German Neonatal Network. Pediatr Infect Dis J 2014; 33:238-43.

5. Brodie SB, Sands KE, Gray JE, et al. Occurrence of nosocomial bloodstream infections in six neonatal intensive care units. Pediatr Infect Dis J 2000; 19:56-65.

6. Chien LY, Macnab Y, Aziz K, et al; Canadian Neonatal Network. Variation in central venous catheter-related infection risks among Canadian neonatal intensive care units. Pediatr Infect Dis J 2002; 21:505-11.

7. Carrieri MP, Stolfi I, Moro MI; Italian Study Group on Hospital Acquired Infections in Neonatal Intensive Care Units. Intercenter variability and time of onset: two crucial issues in the analysis of risk factors for nosocomial sepsis. Pediatr Infect Dis J 2003; 22:599-609.

8. Ballard JL, Khoury JC, Wedig K, et al. New Ballard Score, expanded to include extremely premature infants. J Pediatr 1991; 119:417-23.

9. Kitchen $\mathrm{WH}$, Robinson HP, Dickinson AJ. Revised intrauterine growth curves for an Australian hospital population. Aust Paediatr J 1983; 19:157-61.

10. Bell MJ, Ternberg JL, Feigin RD, et al. Neonatal necrotizing enterocolitis. Therapeutic decisions based upon clinical staging. Ann Surg 1978; 187:1-7.

11. Hornik CP, Fort P, Clark RH, et al. Early and late onset sepsis in very-lowbirth-weight infants from a large group of neonatal intensive care units. Early Hum Dev 2012; 88 Suppl 2:S69-74.

12. Makhoul IR, Sujov P, Smolkin T, Lusky A, Reichman B. Epidemiological, clinical, and microbiological characteristics of late-onset sepsis among very low birth weight infants in Israel: a national survey. Pediatrics 2002; 109:34-9.

13. Klinger G, Levy I, Sirota L, et al; Israel Neonantal Network. Outcome of early-onset sepsis in a national cohort of very low birth weight infants. Pediatrics 2010; 125:e736-40.

14. Tosson AM, Speer CP. Microbial pathogens causative of neonatal sepsis in Arabic countries. J Matern Fetal Neonatal Med 2011; 24:990-4.

15. Stoll BJ, Hansen N, Fanaroff AA, et al. Changes in pathogens causing early-onset sepsis in very-low-birth-weight infants. N Engl J Med 2002; 347:240-7.

16. Lim WH, Lien R, Huang YC, et al. Prevalence and pathogen distribution of neonatal sepsis among very-low-birth-weight infants. Pediatr Neonatol 2012; 53:228-34.

17. Matsubara K, Hoshina K, Suzuki Y. Early-onset and late-onset group B streptococcal disease in Japan: a nationwide surveillance study, 20042010. Int J Infect Dis 2013; 17:e379-84.

18. Klinger G, Levy I, Shirota L, et al; Israel Neonatal Network. Epidemiology and risk factors for early onset sepsis among very-low-birthweight infants. Am J Obstet Gynecol 2009; 201:38.e1-6.

19. Wu JH, Chen CY, Tsao PN, Hsieh WS, Chou HC. Neonatal sepsis: a 6-year analysis in a neonatal care unit in Taiwan. Pediatr Neonatal 2009; 50:8895.

20. Boghossian NS, Page GP, Bell EF, et al; Eunice Kennedy Shriver National Institute of Child Health and Human Development Neonatal Research Network. Late-onset sepsis in very low birth weight infants from singleton and multiple-gestation births. J Pediatr 2013; 162:1120-4, 1124.e1.

21. Stoll BJ, Hansen NI, Sánchez PJ, et al; Eunice Kennedy Shriver National Institute of Child Health and Human Development Neonatal Research Network. Early onset neonatal sepsis: the burden of group B Streptococcal and E. coli disease continues. Pediatrics 2011; 127:817-26.

22. Kaufman D, Fairchild KD. Clinical microbiology of bacterial and fungal sepsis in very-low-birth-weight infants. Clin Microbiol Rev 2004; 17:638-80. 